\title{
Cyclic Contractions and Fixed Point Results via Control Functions on Partial Metric Spaces
}

\author{
Hemant Kumar Nashine ${ }^{1}$ and Zoran Kadelburg ${ }^{2}$ \\ ${ }^{1}$ Department of Mathematics, Disha Institute of Management and Technology, Satya Vihar, \\ Vidhansabha-Chandrakhuri Marg, Mandir Hasaud, Raipur, Chhattisgarh 492101, India \\ ${ }^{2}$ Faculty of Mathematics, University of Belgrade, Studentski trg 16, 11000 Beograd, Serbia \\ Correspondence should be addressed to Zoran Kadelburg; kadelbur@matf.bg.ac.rs
}

Received 23 August 2012; Revised 31 October 2012; Accepted 14 November 2012

Academic Editor: Seenith Sivasundaram

Copyright (C) 2013 H. K. Nashine and Z. Kadelburg. This is an open access article distributed under the Creative Commons Attribution License, which permits unrestricted use, distribution, and reproduction in any medium, provided the original work is properly cited.

Cyclic weaker-type contraction conditions involving a generalized control function (with two variables) are used for mappings on 0-complete partial metric spaces to obtain fixed point results, thus generalizing several known results. Various examples are presented showing how the obtained theorems can be used and that they are proper extensions of the known ones.

\section{Introduction}

The celebrated Banach contraction principle has been generalized in several directions and widely used to obtain various fixed point results, with applications in many branches of mathematics.

Cyclic representations and cyclic contractions were introduced by Kirk et al. [1] and further used by several authors to obtain various fixed point results. See, for example, papers [2-9]. Note that while a classical contraction has to be continuous, cyclic contractions might not be.

On the other hand, Matthews [10] introduced the notion of a partial metric space as a part of the study of denotational semantics of dataflow networks. In partial metric spaces, selfdistance of an arbitrary point need not be equal to zero. Several authors obtained many useful fixed point results in these spaces-we just mention [11-27]. Several results in ordered partial metric spaces have been obtained as well [2836]. Some results for cyclic contractions in partial metric spaces have been very recently obtained in [37-41].

Khan et al. [42] addressed a new category of fixed point problems for a single self-map with the help of a control function which they called an altering distance function. This idea was further used in many papers, such as Choudhury [43] where generalized control functions were used. This approach has been very recently used in $[44,45]$ to obtain fixed point results in partial metric spaces.

In this paper, we extend these results further, considering cyclic weaker-type contraction conditions involving a generalized control function (with two variables) for mappings on 0 -complete partial metric spaces (Romaguera [16]). We obtain fixed point theorems for such mappings, thus generalizing several known results. Various examples are presented showing how the obtained results can be used and that they are proper extensions of the known ones.

\section{Preliminaries}

In 2003, Kirk et al. introduced the following notion of cyclic representation.

Definition 1 (see [1]). Let $X$ be a nonempty set, $m \in \mathbb{N}$, and let $f: X \rightarrow X$ be a self-mapping. Then $X=\bigcup_{i=1}^{m} A_{i}$ is a cyclic representation of $X$ with respect to $f$ if

(a) $A_{i}, i=1, \ldots, m$ are nonempty subsets of $X$;

(b) $f\left(A_{1}\right) \subset A_{2}, f\left(A_{2}\right) \subset A_{3}, \ldots, f\left(A_{m-1}\right) \subset A_{m}$, $f\left(A_{m}\right) \subset A_{1}$.

They proved the following fixed point result. 
Theorem 2 (see [1]). Let $(X, d)$ be a complete metric space, $f: X \rightarrow X$, and let $X=\bigcup_{i=1}^{m} A_{i}$ be a cyclic representation of $X$ with respect to $f$. Suppose that $f$ satisfies the following condition:

$$
\begin{aligned}
& d(f x, f y) \leq \psi(d(x, y)), \\
& \forall x \in A_{i}, \quad y \in A_{i+1}, \quad i \in\{1,2, \ldots, m\},
\end{aligned}
$$

where $A_{m+1}=A_{1}$ and $\psi:[0,1) \rightarrow[0,1)$ is a function, upper semicontinuous from the right and $0 \leq \psi(t)<t$ for $t>0$. Then, $f$ has a fixed point $z \in \bigcap_{i=1}^{m} A_{i}$.

In 2010, Păcurar and Rus introduced the following notion of cyclic weaker $\varphi$-contraction.

Definition 3 (see [2]). Let $(X, d)$ be a metric space, $m \in N$, and let $A_{1}, A_{2}, \ldots, A_{m}$ be closed nonempty subsets of $X$ and $X=\bigcup_{i=1}^{m} A_{i}$. An operator $f: X \rightarrow X$ is called a cyclic weaker $\varphi$-contraction if

(1) $X=\bigcup_{i=1}^{m} A_{i}$ is a cyclic representation of $X$ with respect to $f$;

(2) there exists a continuous, nondecreasing function $\varphi$ : $[0,1) \rightarrow[0,1)$ with $\varphi(t)>0$ for $t \in(0,1)$ and $\varphi(0)=$ 0 such that

$$
d(f x, f y) \leq d(x, y)-\varphi(d(x, y)),
$$

for any $x \in A_{i}, y \in A_{i+1}, i=1,2, \ldots, m$, where $A_{m+1}=A_{1}$.

They proved the following result.

Theorem 4 (see [2]). Suppose that $f$ is a cyclic weaker $\varphi$ contraction on a complete metric space $(X, d)$. Then, $f$ has a fixed point $z \in \bigcap_{i=1}^{m} A_{i}$.

This was generalized by Karapınar in [3].

Khan et al. introduced the following notion.

Definition 5 (see [42]). A function $\varphi:[0,+\infty) \rightarrow[0,+\infty)$ is called an altering distance function if the following properties are satisfied:

(a) $\varphi$ is continuous and nondecreasing,

(b) $\varphi(t)=0 \Leftrightarrow t=0$.

Choudhury introduced a generalization of Chatterjea type contraction as follows.

Definition 6 (see [43]). A self-mapping $T: X \rightarrow X$, on a metric space $(X, d)$, is said to be a weakly $C$-contractive (or a weak Chatterjea type contraction) if for all $x, y \in X$,

$$
\begin{aligned}
d(T x, T y) \leq & \frac{1}{2}[d(x, T y)+d(y, T x)] \\
& -\psi(d(x, T y), d(y, T x)),
\end{aligned}
$$

where $\psi:[0,+\infty)^{2} \rightarrow[0,+\infty)$ is a continuous function such that

$$
\psi(x, y)=0 \quad \text { iff } x=y=0
$$

In [43], the author proved that every weak Chatterjea type contraction on a complete metric space has a unique fixed point.

The following definitions and details can be seen, for example, in $[10,12,13,15,16]$.

Definition 7. A partial metric on a nonempty set $X$ is a function $p: X \times X \rightarrow \mathbb{R}^{+}$such that for all $x, y, z \in X$ :

$$
\begin{aligned}
& \left(p_{1}\right) x=y \Leftrightarrow p(x, x)=p(x, y)=p(y, y), \\
& \left(p_{2}\right) p(x, x) \leq p(x, y), \\
& \left(p_{3}\right) p(x, y)=p(y, x), \\
& \left(p_{4}\right) p(x, y) \leq p(x, z)+p(z, y)-p(z, z) .
\end{aligned}
$$

The pair $(X, p)$ is called a partial metric space.

It is clear that, if $p(x, y)=0$, then from $\left(p_{1}\right)$ and $\left(p_{2}\right) x=$ $y$. But if $x=y, p(x, y)$ may not be 0 .

Each partial metric $p$ on $X$ generates a $T_{0}$ topology $\tau_{p}$ on $X$ which has as a base the family of open $p$-balls $\left\{B_{p}(x, \varepsilon)\right.$ : $x \in X, \varepsilon>0\}$, where $B_{p}(x, \varepsilon)=\{y \in X: p(x, y)<p(x, x)+\varepsilon\}$ for all $x \in X$ and $\varepsilon>0$.

A sequence $\left\{x_{n}\right\}$ in $(X, p)$ converges to a point $x \in X$ (in the sense of $\left.\tau_{p}\right)$ if $\lim _{n \rightarrow \infty} p\left(x, x_{n}\right)=p(x, x)$. This will be denoted as $x_{n} \rightarrow x(n \rightarrow \infty)$ or $\lim _{n \rightarrow \infty} x_{n}=x$. Clearly, a limit of a sequence in a partial metric space need not be unique. Moreover, the function $p(\cdot, \cdot)$ need not be continuous in the sense that $x_{n} \rightarrow x$ and $y_{n} \rightarrow y$ imply $p\left(x_{n}, y_{n}\right) \rightarrow$ $p(x, y)$.

Example 8 (see [10]). (1) A paradigmatic example of a partial metric space is the pair $\left(\mathbb{R}^{+}, p\right)$, where $p(x, y)=\max \{x, y\}$ for all $x, y \in \mathbb{R}^{+}$.

(2) Let $X=\{[a, b]: a, b \in \mathbb{R}, a \leq b\}$ and let $p([a, b],[c, d])=\max \{b, d\}-\min \{a, c\}$. Then $(X, p)$ is a partial metric space.

Definition 9. Let $(X, p)$ be a partial metric space. Then consider the following.

(1) A sequence $\left\{x_{n}\right\}$ in $(X, p)$ is called a Cauchy sequence if $\lim _{n, m \rightarrow \infty} p\left(x_{n}, x_{m}\right)$ exists (and is finite). The space $(X, p)$ is said to be complete if every Cauchy sequence $\left\{x_{n}\right\}$ in $X$ converges, with respect to $\tau_{p}$, to a point $x \in$ $X$ such that $p(x, x)=\lim _{n, m \rightarrow \infty} p\left(x_{n}, x_{m}\right)$.

(2) (see [16]) A sequence $\left\{x_{n}\right\}$ in $(X, p)$ is called 0-Cauchy if $\lim _{n, m \rightarrow \infty} p\left(x_{n}, x_{m}\right)=0$. The space $(X, p)$ is said to be 0 -complete if every 0 -Cauchy sequence in $X$ converges (in $\tau_{p}$ ) to a point $x \in X$ such that $p(x, x)=$ 0 .

Lemma 10. Let $(X, p)$ be a partial metric space.

(a) (see [46, 47]) If $p\left(x_{n}, z\right) \rightarrow p(z, z)=0$ as $n \rightarrow \infty$, then $p\left(x_{n}, y\right) \rightarrow p(z, y)$ as $n \rightarrow \infty$ for each $y \in X$.

(b) (see [16]) If $(X, p)$ is complete, then it is 0-complete.

The converse assertion of (b) does not hold as the following easy example shows. 
Example 11 (see [16]). The space $X=[0,+\infty) \cap \mathbb{Q}$ with the partial metric $p(x, y)=\max \{x, y\}$ is 0 -complete, but is not complete. Moreover, the sequence $\left\{x_{n}\right\}$ with $x_{n}=1$ for each $n \in \mathbb{N}$ is a Cauchy sequence in $(X, p)$, but it is not a 0 -Cauchy sequence.

It is easy to see that every closed subset of a 0 -complete partial metric space is 0 -complete.

\section{Main Results}

In this section, we will prove some fixed point theorems for self-mappings defined on a 0 -complete partial metric space and satisfying certain cyclic weak contractive condition involving a generalized control function. To achieve our goal, we introduce the new notion of a cyclic contraction.

Definition 12. Let $(X, p)$ be a partial metric space, $q \in \mathbb{N}$, and let $A_{1}, A_{2}, \ldots, A_{q}$ be nonempty subsets of $X$ and $Y=\bigcup_{i=1}^{q} A_{i}$. An operator $T: Y \rightarrow Y$ is called a cyclic contraction under weak contractive condition if:

(NZ1) $Y=\bigcup_{i=1}^{q} A_{i}$ is a cyclic representation of $Y$ with respect to $T$;

(NZ2) for any $(x, y) \in A_{i} \times A_{i+1}, i=1,2, \ldots, q\left(\right.$ with $A_{q+1}=$ $\left.A_{1}\right)$,

$$
p(T x, T y) \leq M(x, y)-\psi(p(x, y), p(x, T x)),
$$

where

$$
\begin{aligned}
M(x, y)=\max \{p(x, y), p(x, T x), p(y, T y), & \\
& \left.\frac{1}{2}[p(x, T y)+p(T x, y)]\right\},
\end{aligned}
$$

and $\psi:[0, \infty)^{2} \rightarrow[0, \infty)$ is a lower semicontinuous mapping such that $\psi(s, t)=0$ if and only if $s=t=0$.

Our main result is the following.

Theorem 13. Let $(X, p)$ be a 0-complete partial metric space, let $q \in \mathbb{N}, A_{1}, A_{2}, \ldots, A_{q}$ be nonempty closed subsets of $(X, p)$, and let $Y=\bigcup_{i=1}^{q} A_{i}$. Suppose that $T: Y \rightarrow Y$ is a cyclic contraction as defined in Definition 12. Then, $T$ has a unique fixed point $z \in Y$ such that $p(z, z)=0$. Moreover, $z \in \bigcap_{i=1}^{q} A_{i}$. Each Picard sequence $x_{n}=T^{n} x_{0}, x_{0} \in Y$ converges to $z$ in topology $\tau_{p}$.

Proof. Let $x_{0}$ be an arbitrary point of $Y$. Then there exists some $i_{0}$ such that $x_{0} \in A_{i_{0}}$. Now $x_{1}=T x_{0} \in A_{i_{0}+1}$ and, similarly, $x_{n}:=T x_{n-1}=T^{n} x_{0} \in A_{i_{0}+n}$ for $n \in \mathbb{N}$, where $A_{q+k}=A_{k}$. In the case $p\left(x_{n_{0}}, x_{n_{0}+1}\right)=0$ for some $n_{0} \in \mathbb{N}_{0}$, it is clear that $x_{n_{0}}$ is a fixed point of $T$.

Without loss of the generality, we may assume that

$$
p\left(x_{n}, x_{n+1}\right)>0, \quad \forall n \in \mathbb{N} .
$$

From the condition (NZ1), we observe that for all $n$, there exists $i=i(n) \in\{1,2, \ldots, q\}$ such that $\left(x_{n}, x_{n+1}\right) \in A_{i} \times A_{i+1}$.
Putting $x=x_{n}$ and $y=x_{n+1}$ in (NZ2) condition, we have

$$
\begin{aligned}
p\left(x_{n+1}, x_{n+2}\right)= & p\left(T x_{n}, T x_{n+1}\right) \\
\leq & M\left(x_{n}, x_{n+1}\right)-\psi\left(p\left(x_{n}, x_{n+1}\right), p\left(x_{n}, T x_{n}\right)\right) \\
= & \max \left\{p\left(x_{n}, x_{n+1}\right), p\left(x_{n+1}, x_{n+2}\right),\right. \\
& \left.\frac{1}{2}\left[p\left(x_{n}, x_{n+2}\right)+p\left(x_{n+1}, x_{n+1}\right)\right]\right\} \\
& -\psi\left(p\left(x_{n}, x_{n+1}\right), p\left(x_{n}, x_{n+1}\right)\right) .
\end{aligned}
$$

By $\left(p_{4}\right)$, we have

$$
p\left(x_{n}, x_{n+2}\right)+p\left(x_{n+1}, x_{n+1}\right) \leq p\left(x_{n}, x_{n+1}\right)+p\left(x_{n+1}, x_{n+2}\right) .
$$

Therefore,

$$
\begin{aligned}
\max & \left\{p\left(x_{n}, x_{n+1}\right), p\left(x_{n+1}, x_{n+2}\right),\right. \\
& \left.\frac{1}{2}\left[p\left(x_{n}, x_{n+2}\right)+p\left(x_{n+1}, x_{n+1}\right)\right]\right\} \\
& \leq \max \left\{p\left(x_{n}, x_{n+1}\right), p\left(x_{n+1}, x_{n+2}\right)\right\} .
\end{aligned}
$$

By (8) and (10), we have

$$
\begin{aligned}
p\left(x_{n+1}, x_{n+2}\right) \leq & \max \left\{p\left(x_{n}, x_{n+1}\right), p\left(x_{n+1}, x_{n+2}\right)\right\} \\
& -\psi\left(p\left(x_{n}, x_{n+1}\right), p\left(x_{n}, x_{n+1}\right)\right) .
\end{aligned}
$$

If $\max \left\{p\left(x_{n}, x_{n+1}\right), p\left(x_{n+1}, x_{n+2}\right)\right\}=p\left(x_{n+1}, x_{n+2}\right)$, then from (11), we have

$$
\begin{aligned}
& p\left(x_{n+1}, x_{n+2}\right) \\
& \quad \leq p\left(x_{n+1}, x_{n+2}\right)-\psi\left(p\left(x_{n}, x_{n+1}\right), p\left(x_{n}, x_{n+1}\right)\right) \\
& \quad<p\left(x_{n+1}, x_{n+2}\right)
\end{aligned}
$$

which is a contradiction (it was used that $\psi\left(p\left(x_{n}, x_{n+1}\right)\right.$, $\left.p\left(x_{n}, x_{n+1}\right)\right)>0$ since $\left.p\left(x_{n}, x_{n+1}\right)>0\right)$. Hence, $p\left(x_{n}, x_{n+1}\right)=$ 0 , and $x_{n}=x_{n+1}$, which is excluded. Therefore, we have $\max \left\{p\left(x_{n}, x_{n+1}\right), p\left(x_{n+1}, x_{n+2}\right)\right\}=p\left(x_{n}, x_{n+1}\right)$ and hence

$$
\begin{aligned}
& p\left(x_{n+1}, x_{n+2}\right) \\
& \quad \leq p\left(x_{n}, x_{n+1}\right)-\psi\left(p\left(x_{n}, x_{n+1}\right), p\left(x_{n}, x_{n+1}\right)\right) \\
& \quad \leq p\left(x_{n}, x_{n+1}\right) .
\end{aligned}
$$

By (13), we have that $\left\{p\left(x_{n}, x_{n+1}\right)\right\}$ is a nonincreasing sequence of positive real numbers. Thus, there exists $r \geq 0$ such that

$$
\lim _{n \rightarrow \infty} p\left(x_{n}, x_{n+1}\right)=r
$$


Passing to the limit as $n \rightarrow \infty$ in (13) and using (14) and lower semicontinuity of $\psi$, we have

$$
\begin{aligned}
r & \leq r-\liminf _{n \rightarrow \infty} \psi\left(p\left(x_{n}, x_{n+1}\right), p\left(x_{n}, x_{n+1}\right)\right) \\
& \leq r-\psi(r, r)
\end{aligned}
$$

thus, $\psi(r, r)=0$ and hence $r=0$. Therefore

$$
\lim _{n \rightarrow \infty} p\left(x_{n}, x_{n+1}\right)=0 .
$$

Next, we claim that $\left\{x_{n}\right\}$ is a 0 -Cauchy sequence in the space $(X, p)$. Suppose that this is not the case. Then there exists $\varepsilon>0$ for which we can find two sequences of positive integers $\{m(k)\}$ and $\{n(k)\}$ such that for all positive integers $k$

$$
\begin{gathered}
n(k)>m(k)>k, \\
p\left(x_{m(k)}, x_{n(k)}\right) \geq \varepsilon, \\
p\left(x_{m(k)}, x_{n(k)-1}\right)<\varepsilon .
\end{gathered}
$$

Using (17) and $\left(p_{4}\right)$, we get

$$
\begin{aligned}
\varepsilon \leq & p\left(x_{n(k)}, x_{m(k)}\right) \\
\leq & p\left(x_{m(k)}, x_{n(k)-1}\right) \\
& +p\left(x_{n(k)-1}, x_{n(k)}\right)-p\left(x_{n(k)-1}, x_{n(k)-1}\right) \\
& <\varepsilon+p\left(x_{n(k)}, x_{n(k)-1}\right) .
\end{aligned}
$$

Thus we have

$$
\varepsilon \leq p\left(x_{n(k)}, x_{m(k)}\right)<\varepsilon+p\left(x_{n(k)}, x_{n(k)-1}\right) .
$$

Passing to the limit as $k \rightarrow \infty$ in the above inequality and using (16), we obtain

$$
\lim _{k \rightarrow \infty} p\left(x_{n(k)}, x_{m(k)}\right)=\varepsilon .
$$

On the other hand, for all $k$, there exists $j(k) \in\{1, \ldots, q\}$ such that $n(k)-m(k)+j(k) \equiv 1[q]$. Then $x_{m(k)-j(k)}$ (for $k$ large enough, $m(k)>j(k))$ and $x_{n(k)}$ lie in different adjacently labelled sets $A_{i}$ and $A_{i+1}$ for certain $i \in\{1, \ldots, q\}$.

Using $\left(p_{4}\right)$ and (20), we get

$$
\begin{aligned}
p\left(x_{m(k)-j(k)}, x_{n(k)}\right) & \\
\leq & p\left(x_{m(k)-j(k)}, x_{m(k)}\right) \\
& +p\left(x_{n(k)}, x_{m(k)}\right)-p\left(x_{n(k)}, x_{n(k)}\right) \\
\leq & \sum_{l=0}^{j(k)-1} p\left(x_{m(k)-j(k)+l}, x_{m(k)-j(k)+l+1}\right)+p\left(x_{n(k)}, x_{m(k)}\right) \\
\leq & \sum_{l=0}^{q-1} p\left(x_{m(k)-j(k)+l}, x_{m(k)-j(k)+l+1}\right) \\
& +p\left(x_{n(k)}, x_{m(k)}\right) \longrightarrow \varepsilon \text { as } k \longrightarrow \infty \text { (from (16)), }
\end{aligned}
$$

that is,

$$
\lim _{k \rightarrow \infty} p\left(x_{m(k)-j(k)}, x_{n(k)}\right)=\varepsilon .
$$

Using (16), we have

$$
\begin{gathered}
\lim _{k \rightarrow \infty} p\left(x_{m(k)-j(k)+1}, x_{m(k)-j(k)}\right)=0, \\
\lim _{k \rightarrow \infty} p\left(x_{n(k)+1}, x_{n(k)}\right)=0 .
\end{gathered}
$$

Again, using $\left(p_{4}\right)$, we get

$$
\begin{aligned}
& p\left(x_{m(k)-j(k)}, x_{n(k)+1}\right) \leq p\left(x_{m(k)-j(k)}, x_{n(k)}\right) \\
& \quad+p\left(x_{n(k)}, x_{n(k)+1}\right)-p\left(x_{n(k)}, x_{n(k)}\right) .
\end{aligned}
$$

Passing to the limit as $k \rightarrow \infty$ in the pervious inequality, and using (24) and (22), we get

$$
\lim _{k \rightarrow \infty} p\left(x_{m(k)-j(k)}, x_{n(k)+1}\right)=\varepsilon .
$$

Similarly, we have by $\left(p_{4}\right)$

$$
\begin{aligned}
& p\left(x_{n(k)}, x_{m(k)-j(k)+1}\right) \\
& \leq p\left(x_{m(k)-j(k)}, x_{m(k)-j(k)+1}\right) \\
& \quad+p\left(x_{m(k)-j(k)}, x_{n(k)}\right)-p\left(x_{m(k)-j(k)}, x_{m(k)-j(k)}\right) .
\end{aligned}
$$

Passing to the limit as $k \rightarrow \infty$, and using (16) and (22), we obtain

$$
\lim _{k \rightarrow \infty} p\left(x_{n(k)}, x_{m(k)-j(k)+1}\right)=\varepsilon .
$$

Similarly, we have by $\left(p_{4}\right)$

$$
\lim _{k \rightarrow \infty} p\left(x_{m(k)-j(k)+1}, x_{n(k)+1}\right)=\varepsilon .
$$

Using (NZ2), we obtain

$$
\begin{gathered}
p\left(x_{m(k)-j(k)+1}, x_{n(k)+1}\right) \\
\leq \max \left\{p\left(x_{m(k)-j(k)}, x_{n(k)}\right), p\left(x_{m(k)-j(k)+1}, x_{m(k)-j(k)}\right)\right. \\
p\left(x_{n(k)+1}, x_{n(k)}\right), \frac{1}{2} p\left(x_{m(k)-j(k)}, x_{n(k)+1}\right) \\
\left.+p\left(x_{n(k)}, x_{m(k)-j(k)+1}\right)\right\} \\
-\psi\left(p\left(x_{m(k)-j(k)}, x_{n(k)}\right), p\left(x_{m(k)-j(k)+1}, x_{m(k)-j(k)}\right)\right)
\end{gathered}
$$

for all $k$. Passing to the limit as $k \rightarrow \infty$ in the last inequality (and using the lower semicontinuity of the function $\psi$ ), we obtain

$$
\begin{aligned}
\varepsilon \leq \varepsilon-\liminf _{n \rightarrow \infty} \psi( & p\left(x_{m(k)-j(k)}, x_{n(k)}\right), \\
& \left.p\left(x_{m(k)-j(k)+1}, x_{m(k)-j(k)}\right)\right)
\end{aligned}
$$

$$
\leq \varepsilon-\psi(\varepsilon, 0),
$$


which implies that $\psi(\varepsilon, 0)=0$; that is a contradiction since $\varepsilon>0$. We deduce that $\left\{x_{n}\right\}$ is a 0 -Cauchy sequence.

Since $(X, p)$ is 0 -complete and $Y$ is closed, it follows that the sequence $\left\{x_{n}\right\}$ converges to some $u \in Y$, that is:

$$
\lim _{n \rightarrow \infty} x_{n}=u
$$

We shall prove that

$$
u \in \bigcap_{i=1}^{q} A_{i} .
$$

From condition (NZ1), and since $x_{0} \in A_{1}$, we have $\left\{x_{n q}\right\}_{n \geq 0} \subseteq$ $A_{1}$. Since $A_{1}$ is closed, from (32), we get that $u \in A_{1}$. Again, from the condition (NZ1), we have $\left\{x_{n q+1}\right\}_{n \geq 0} \subseteq A_{2}$. Since $A_{2}$ is closed, from (32), we get that $u \in A_{2}$. Continuing this process, we obtain $(33)$ and $p(u, u)=0$.

Now, we shall prove that $u$ is a fixed point of $T$. Indeed, from (33), since for all $n$, there exists $i(n) \in\{1,2, \ldots, q\}$ such that $x_{n} \in A_{i(n)}$; applying (NZ2) with $x=u$ and $y=x_{n}$, we obtain

$$
\begin{aligned}
p\left(T u, x_{n+1}\right)= & p\left(T u, T x_{n}\right) \\
\leq & \max \left\{p\left(u, x_{n}\right), p(u, T u), p\left(x_{n}, x_{n+1}\right)\right. \\
& \left.\frac{1}{2} p\left(u, x_{n+1}\right)+p\left(x_{n}, T u\right)\right\} \\
& -\psi\left(p\left(u, x_{n}\right), p(u, T u)\right)
\end{aligned}
$$

for all $n$. Passing to the limit as $n \rightarrow \infty$ in (34), and using (32), we get

$$
\begin{aligned}
p(u, T u) \leq & \lim _{n \rightarrow \infty} \max \left\{p\left(u, x_{n}\right), p(u, T u), p\left(x_{n}, x_{n+1}\right),\right. \\
& \left.\frac{1}{2} p\left(u, x_{n+1}\right)+p\left(x_{n}, T u\right)\right\} \\
& -\liminf _{n \rightarrow \infty} \psi\left(p\left(u, x_{n}\right), p(u, T u)\right) \\
\leq & \max \left\{0, p(u, T u), 0, \frac{1}{2} p(u, T u)\right\} \\
& -\psi(0, p(u, T u))=p(u, T u)-\psi(0, p(u, T u)),
\end{aligned}
$$

which is impossible unless $\psi(0, p(u, T u))=0$, so

$$
u=T u
$$

that is, $u$ is a fixed point of $T$.

We claim that there is a unique fixed point of $T$. Assume on the contrary that $T u=u$ and $T v=v$ with $p(u, v)>0$.
By supposition, we can replace $x$ by $u$ and $y$ by $v$ in (NZ2) to obtain

$$
\begin{aligned}
p(u, v)= & p(T u, T v) \\
\leq & \max \{p(u, v), p(u, T u), p(v, T v), \\
& \left.\frac{1}{2}[p(u, T v)+p(T u, v)]\right\} \\
& -\psi(p(u, v), p(u, T u)) \\
= & p(u, v)-\psi(p(u, v), 0)<p(u, v),
\end{aligned}
$$

a contradiction. Hence $p(u, v)=0$, that is, $u=v$. We conclude that $T$ has only one fixed point in $X$. The proof is complete.

If we take $q=1$ and $A_{1}=X$ in Theorem 13, then we get the following fixed point theorem.

Corollary 14. Let $(X, p)$ be a 0 -complete partial metric space, and let $T: X \rightarrow X$ satisfy the following condition: there exists a lower semicontinuous mapping $\psi:[0, \infty)^{2} \rightarrow[0, \infty)$ such that $\psi(t, s)=0$ if and only if $t=s=0$ and that

$$
\begin{aligned}
& p(T x, T y) \leq \max \{p(x, y), p(x, T x), p(y, T y), \\
&\left.\frac{1}{2}[p(x, T y)+p(y, T x)]\right\} \\
&-\psi(p(x, y), p(x, T x)),
\end{aligned}
$$

for all $x, y \in X$. Then $T$ has a unique fixed point $z \in X$. Moreover, $p(z, z)=0$.

Corollary 14 extends and generalizes many existing fixed point theorems in the literature.

By taking $\psi(s, t)=(1-r) \max \{s, t\}$ where $r \in[0,1)$ in Theorem 13, we have the following result.

Corollary 15. Let $(X, p)$ be a 0 -complete partial metric space, let $q \in \mathbb{N}, A_{1}, A_{2}, \ldots, A_{q}$ be nonempty closed subsets of $X$, $Y=\bigcup_{i=1}^{q} A_{i}$, and $T: Y \rightarrow Y$ such that

(NZ1) $Y=\bigcup_{i=1}^{q} A_{i}$ is a cyclic representation of $Y$ with respect to $T$;

(NZ3) for any $(x, y) \in A_{i} \times A_{i+1}, \quad i=1,2, \ldots, q\left(\right.$ with $A_{q+1}=$ $\left.A_{1}\right)$,

$$
\begin{array}{r}
p(T x, T y) \leq r \max \{p(x, y), p(x, T x), p(y, T y), \\
\left.\frac{1}{2}[p(x, T y)+p(T x, y)]\right\},
\end{array}
$$

where $r \in[0,1)$. Then $T$ has a unique fixed point $z$ belonging to $\bigcap_{i=1}^{q} A_{i}$; moreover, $p(z, z)=0$.

As a special case of Corollary 15, we obtain Matthews's version of Banach contraction principle [10]. 
Corollary 16. Let $(X, p)$ be a 0 -complete partial metric space, let $p \in \mathbb{N}, A_{1}, A_{2}, \ldots, A_{q}$ be nonempty closed subsets of $X$, $Y=\bigcup_{i=1}^{q} A_{i}$, and $T: Y \rightarrow Y$ such that

(NZ1) $X=\bigcup_{i=1}^{q} A_{i}$ is a cyclic representation of $X$ with respect to $T$;

(NZ4) for any $(x, y) \in A_{i} \times A_{i+1}, \quad i=1,2, \ldots, q\left(\right.$ with $A_{q+1}=$ $\left.A_{1}\right)$,

$$
\begin{gathered}
p\left(T^{n} x, T^{n} y\right) \leq \max \left\{p(x, y), p\left(x, T^{n} x\right), p\left(y, T^{n} y\right),\right. \\
\left.\frac{1}{2}\left[p\left(x, T^{n} y\right)+p\left(y, T^{n} x\right)\right]\right\} \\
-\psi\left(p(x, y), p\left(x, T^{n} x\right)\right),
\end{gathered}
$$

where $n$ is a positive integer and $\psi:[0, \infty)^{2} \rightarrow[0, \infty)$ is a lower semi-continuous mapping such that $\psi(t, s)=0$ if and only if $t=s=0$. Then $T$ has a unique fixed point belonging to $\bigcap_{i=1}^{q} A_{i}$.

\section{Examples}

The following example shows how Theorem 13 can be used. It is adapted from [38, Example 2.9].

Example 17. Consider the partial metric space $(X, d)$ of Example 8 (2). It is easy to see that it is 0 -complete. Consider the following closed subsets of $X$ :

$$
\begin{aligned}
& A_{1}=\left\{\left[1-2^{-n}, 1\right]: n \in \mathbb{N}\right\} \cup\{\{1\}\}, \\
& A_{2}=\left\{\left[1,1+2^{-n}\right]: n \in \mathbb{N}\right\} \cup\{\{1\}\},
\end{aligned}
$$

$Y=A_{1} \cup A_{2}$, and define a mapping $T: Y \rightarrow Y$ by

$$
T x= \begin{cases}{\left[1,1+2^{-(n+1)}\right],} & \text { if } x=\left[1-2^{-n}, 1\right], \\ {\left[1-2^{-(n+1)}, 1\right],} & \text { if } x=\left[1,1+2^{-n}\right], \\ \{1\}, & \text { if } x=\{1\} .\end{cases}
$$

Obviously, $Y=A_{1} \cup A_{2}$ is a cyclic representation of $Y$ with respect to $T$. We will show that $T$ satisfies the contractive condition (NZ2) of Definition 12 with the control function $\psi:[0,+\infty)^{2} \rightarrow[0,+\infty)$ given by $\psi(s, t)=(1 / 2) \max \{s, t\}$

Let $(x, y) \in A_{1} \times A_{2}$ (the other possibility is treated similarly) and consider the following cases:

(1) $x=\left[1-2^{-n}, 1\right], y=\left[1,1+2^{-k}\right]$ and $n<k$, that is, $n+1 \leq k$. Then $p(T x, T y)=p\left(\left[1,1+2^{-(n+1)}\right],[1-\right.$ $\left.\left.2^{-(k+1)}, 1\right]\right)=(1 / 2)\left(2^{-n}+2^{-k}\right) \leq(3 / 4) \cdot 2^{-n}$,

$$
M(x, y)=\max \left\{2^{-n}+2^{-k}, \frac{3}{2} \cdot 2^{-n}, \frac{3}{2} \cdot 2^{-k}\right. \text {, }
$$

$$
\left.\frac{1}{2}\left(\left|2^{-n}-2^{-(k+1)}\right|+\left|2^{-k}-2^{-(n+1)}\right|\right)\right\}
$$

$$
=\frac{3}{2} \cdot 2^{-n}
$$

and $\psi(p(x, y), p(x, T x))=(1 / 2) \cdot(3 / 2) \cdot 2^{-n}=(3 / 4)$. $2^{-n}$. Hence, the condition (NZ2) reduces to $(3 / 4)$. $2^{-n} \leq(3 / 2) \cdot 2^{-n}-(3 / 4) \cdot 2^{-n}$ and holds true.

(2) $x=\left[1-2^{-n}, 1\right], y=\left[1,1+2^{-k}\right]$ and $n=k$. Then $p(T x, T y)=2^{-n}, M(x, y)=2 \cdot 2^{-n}$, and $\psi(p(x, y), p(x, T x))=(1 / 2) \cdot 2 \cdot 2^{-n}=2^{-n}$; hence (NZ2) reduces to $2^{-n} \leq 2 \cdot 2^{-n}-2^{-n}$.

(3) $x=\left[1-2^{-n}, 1\right], y=\left[1,1+2^{-k}\right]$ and $n>k$, that is, $n \geq k+1$. Then $p(T x, T y) \leq(3 / 4) \cdot 2^{-k}, M(x, y)=$ $(3 / 2) \cdot 2^{-k}$ and $\psi(p(x, y), p(x, T x))=(1 / 2) \cdot(3 / 2) \cdot$ $2^{-k}=(3 / 4) \cdot 2^{-k}$. Hence, $(\mathrm{NZ2})$ reduces to $(3 / 4) \cdot 2^{-k} \leq$ $(3 / 2) \cdot 2^{-k}-(3 / 4) \cdot 2^{-k}$ and holds true.

(4) $x=\left[1-2^{-n}, 1\right], y=\{1\}$. Then $p(T x, T y)=p([1,1+$ $\left.\left.2^{-(n+1)}\right],\{1\}\right)=(1 / 2) \cdot 2^{-n}, M(x, y)=p(x, T x)=$ $(3 / 2) \cdot 2^{-n}$ and $\psi(p(x, y), p(x, T x))=(1 / 2) \cdot(3 / 2) \cdot$ $2^{-n}=(3 / 4) \cdot 2^{-n} \cdot(\mathrm{NZ} 2)$ reduces to $(1 / 2) \cdot 2^{-n} \leq$ $(3 / 2) \cdot 2^{-n}-(3 / 4) \cdot 2^{-n}$.

(5) The case $x=\{1\}, y=\left[1,1+2^{-k}\right]$ is treated symmetrically.

(6) The case $x=y=\{1\}$ is trivial.

We conclude that all conditions of Theorem 13 are satisfied. The mapping $T$ has a unique fixed point $\{1\} \in A_{1} \cap$ $A_{2}$.

Here is another example showing the use of Theorem 13.

Example 18. Let $X=[0,1]$ be equipped with the partial metric given as

$$
p(x, y)= \begin{cases}|x-y|, & \text { if } x, y \in[0,1), \\ 1, & \text { if } x=1 \vee y=1 .\end{cases}
$$

Then, $(X, p)$ is a 0 -complete partial metric space. Let $A_{1}=$ $[0,1 / 2], A_{2}=[1 / 2,1]$, and $T: X \rightarrow X$ be given as

$$
T x= \begin{cases}\frac{1}{2}, & \text { if } x \in[0,1), \\ \frac{1}{6}, & \text { if } x=1 .\end{cases}
$$

Obviously, $X=A_{1} \cup A_{2}$ is a cyclic representation of $X$ with respect to $T$. We will check the contractive condition (NZ2) of Definition 12 with the control function $\psi:[0,+\infty)^{2} \rightarrow$ $[0,+\infty)$ given by $\psi(s, t)=(s+t) /(2+s+t)$. Let $(x, y) \in A_{1} \times A_{2}$ (the other possibility is treated symmetrically). Consider the following possible cases.

(1) $x \in[0,1 / 2], y \in[1 / 2,1)$. Then $p(T x, T y)=$ $p(1 / 2,1 / 2)=0, M(x, y)=\max \{y-x, 1 / 2-$ $x, y-1 / 2,(1 / 2)(1 / 2-x+y-1 / 2)\}=y-x$. $\psi(p(x, y), p(x, T x))=(y+1 / 2-2 x) /(5 / 2+y-2 x)$. It is easy to check that

$$
\begin{aligned}
p(T x, T y) & =0 \leq y-x-\frac{y+1 / 2-2 x}{5 / 2+y-2 x} \\
& =M(x, y)-\psi(p(x, y), p(x, T x))
\end{aligned}
$$

holds for the given values of $x$ and $y$. 
(2) $x \in[0,1 / 2], y=1$. Then $p(T x, T y)=p(1 / 2,1 / 6)=$ $1 / 3, M(x, y)=1$ and $\psi(p(x, y), p(x, T x))=$ $\psi(1,1 / 2-x)=(3 / 2-x) /(7 / 2-x)$. The condition (NZ2) reduces to

$$
\frac{1}{3} \leq 1-\frac{3-2 x}{7-2 x}
$$

and can be checked directly.

Thus, all the conditions of Theorem 13 are fulfilled, and we conclude that $T$ has a unique fixed point $1 / 2 \in A_{1} \cap A_{2}$.

We state a more involved example that is inspired with the one from [48].

Example 19. Let $X \subset \ell^{1}, X \ni x=\left(x_{n}\right)_{n=1}^{\infty}$ if and only if $x_{n} \geq 0$ for each $n \in \mathbb{N}$. Define a partial metric $p$ on $X$ by

$$
p\left(\left(x_{n}\right),\left(y_{n}\right)\right)=\sum_{n=1}^{\infty} \max \left\{x_{n}, y_{n}\right\}
$$

(it is easy to check that axioms $\left(p_{1}\right)-\left(p_{4}\right)$ hold true). Let $\alpha \epsilon$ $(0,1)$ be fixed, denote $\mathbf{0}=(0)_{n=1}^{\infty}$, and consider the subsets $A_{1}$ and $A_{2}$ of $X$ defined by $A_{1}=A^{\prime} \cup\{\mathbf{0}\}, A_{2}=A^{\prime \prime} \cup\{\mathbf{0}\}$, where

$$
\begin{aligned}
& A^{\prime} \ni x^{l}=\left(x_{n}^{l}\right)_{n=1}^{\infty} \\
& \text { iff } x_{n}^{l}=\left\{\begin{array}{cc}
0, & n<2 l \vee n=2 k-1, k \in \mathbb{N}, \\
\alpha^{n}, & n=2 k \geq 2 l
\end{array} \quad l=1,2, \ldots,\right. \\
& A^{\prime \prime} \ni x^{l}=\left(x_{n}^{l}\right)_{n=1}^{\infty} \\
& \text { iff } x_{n}^{l}=\left\{\begin{array}{cc}
0, & n<2 l-1 \vee n=2 k, k \in \mathbb{N}, \\
\alpha^{n}, & n=2 k-1 \geq 2 l-1
\end{array} \quad l=1,2, \ldots .\right.
\end{aligned}
$$

Denote $Y=A_{1} \cup A_{2}$ (obviously $A_{1} \cap A_{2}=\{\mathbf{0}\}$ ).

Consider the mapping $T: Y \rightarrow Y$ given by.

$$
\begin{gathered}
T(\mathbf{0})=\mathbf{0}, \\
T\left((\underbrace{0, \ldots, 0}_{2 l-1}, \alpha^{2 l}, 0, \alpha^{2 l+2}, 0, \ldots)\right) \\
=(\underbrace{0, \ldots, 0}_{2 l}, \alpha^{2 l+1}, 0, \alpha^{2 l+3}, 0, \ldots), \\
T\left((\underbrace{0, \ldots, 0}_{2 l}, \alpha^{2 l+1}, 0, \alpha^{2 l+3}, 0, \ldots)\right) \\
=(\underbrace{0, \ldots, 0}_{2 l+1}, \alpha^{2 l+2}, 0, \alpha^{2 l+4}, 0, \ldots) .
\end{gathered}
$$

Obviously, $T\left(A_{1}\right) \subset A_{2}$ and $T\left(A_{2}\right) \subset A_{1}$; hence $Y=A_{1} \cup$ $A_{2}$ is a cyclic representation of $Y$ with respect to $T$. Take $\psi$ : $[0,+\infty)^{2} \rightarrow[0,+\infty)$ defined by $\psi(s, t)=(1-\alpha) \max \{s, t\}$.

Let us check the contractive condition (NZ2) of Theorem 13. Take $x=(\underbrace{0, \ldots, 0}_{2 l-1}, \alpha^{2 l}, 0, \alpha^{2 l+2}, 0, \ldots) \in A_{1}$ and $y=(\underbrace{0, \ldots, 0}_{2 m}, \alpha^{2 m+1}, 0, \alpha^{2 m+3}, 0, \ldots) \in A_{2}$ and assume, for example, that $l \leq m$ (the case $l>m$ is treated similarly, as well as the case when $x$ or $y$ is equal to $\mathbf{0}$ ). Then

$$
\begin{gathered}
p(x, y)=\alpha^{2 l}+\cdots+\alpha^{2 m-2}+\frac{\alpha^{2 m}}{1-\alpha}, \\
p(T x, T y)=\alpha^{2 l+1}+\cdots+\alpha^{2 m-1}+\frac{\alpha^{2 m+1}}{1-\alpha}, \\
p(x, T x)=\frac{\alpha^{2 l}}{1-\alpha}, \quad p(y, T y)=\frac{\alpha^{2 m+1}}{1-\alpha} .
\end{gathered}
$$

Hence,

$$
\begin{aligned}
p(T x, T y) & =\alpha p(x, y) \leq \alpha \cdot \frac{\alpha^{2 l}}{1-\alpha} \\
& =\frac{\alpha^{2 l}}{1-\alpha}-(1-\alpha) \frac{\alpha^{2 l}}{1-\alpha} \\
& =M(x, y)-\psi(p(x, y), p(x, T x))
\end{aligned}
$$

Obviously, $T$ has a unique fixed point $\mathbf{0} \in A_{1} \cap A_{2}$.

Finally, we present an example showing that in certain situations the existence of a fixed point can be concluded under partial metric conditions, while the same cannot be obtained using the standard metric.

Example 20. Let $X=\mathbb{R}^{+}$be equipped with the usual partial metric $p(x, y)=\max \{x, y\}$. Suppose $A_{1}=[0,1], A_{2}=$ $[0,1 / 2], A_{3}=[0,1 / 6], A_{4}=[0,1 / 42]$ and $Y=\bigcup_{i=1}^{4} A_{i}$. Consider the mapping $T: Y \rightarrow Y$ defined by

$$
T x=\frac{x^{2}}{1+x} \text {. }
$$

It is clear that $\bigcup_{i=1}^{4} A_{i}$ is a cyclic representation of $Y$ with respect to $T$. Further, consider the function $\psi:[0,+\infty)^{2} \rightarrow$ $[0,+\infty)$ given by

$$
\psi(s, t)=\frac{s+t}{2+s+t} .
$$

Take an arbitrary pair $(x, y) \in A_{i} \times A_{i+1}$ with, say, $y \leq x$ (the other possibility can be treated in a similar way). Then

$$
p(T x, T y)=\max \left\{\frac{x^{2}}{1+x}, \frac{y^{2}}{1+y}\right\}=\frac{x^{2}}{1+x} .
$$


On the other hand,

$$
\begin{aligned}
M(x, y)=\max \left\{p(x, y), p\left(x, \frac{x^{2}}{1+x}\right), p\left(y, \frac{y^{2}}{1+y}\right),\right. \\
\left.\quad \frac{1}{2}\left(p\left(x, \frac{y^{2}}{1+y}\right)+p\left(y, \frac{x^{2}}{1+x}\right)\right)\right\} \\
=\max \left\{x, x, y, \frac{1}{2}\left(x+\max \left\{y, \frac{x^{2}}{1+x}\right\}\right)\right\}=x, \\
M(x, y)-\psi(p(x, y), p(x, T x))=x-\frac{2 x}{2+2 x}=\frac{x^{2}}{1+x} .
\end{aligned}
$$

Hence, condition (NZ2) is satisfied, as well as other conditions of Theorem 13 (with $q=4$ ). We deduce that $T$ has a unique fixed point $z=0 \in A_{1} \cap A_{2} \cap A_{3} \cap A_{4}$.

On the other hand, consider the same problem in the standard metric $d(x, y)=|x-y|$ and take $x=1$ and $y=1 / 2$. Then

$$
\begin{gathered}
d(T x, T y)=\left|\frac{1}{2}-\frac{1}{6}\right|=\frac{1}{3}, \\
M(x, y)=\max \left\{\frac{1}{2}, \frac{1}{2}, \frac{1}{3}, \frac{1}{2}\left(\frac{5}{6}+0\right)\right\}=\frac{1}{2}
\end{gathered}
$$

and hence

$$
M(x, y)-\psi(d(x, y), d(x, T x))=\frac{1}{2}-\frac{1}{3}=\frac{1}{6} .
$$

Thus, condition (NZ2) for $p=d$ does not hold and the existence of a fixed point of $T$ cannot be derived using the standard metric.

Remark 21. The results of this paper are obtained under the assumption that the given partial metric space is 0-complete. Taking into account Lemma 10 and Example 11, it follows that they also hold if the space is complete, but that our assumption is weaker.

\section{Acknowledgments}

The authors thank the referees for valuable suggestions that helped them to improve the text. The second author is thankful to the Ministry of Science and Technological Development of Serbia.

\section{References}

[1] W. A. Kirk, P. S. Srinivasan, and P. Veeramani, "Fixed points for mappings satisfying cyclical contractive conditions," Fixed Point Theory, vol. 4, no. 1, pp. 79-89, 2003.

[2] M. Păcurar and I. A. Rus, "Fixed point theory for cyclic $\varphi$ contractions," Nonlinear Analysis, vol. 72, no. 3-4, pp. 1181-1187, 2010.

[3] E. Karapınar, "Fixed point theory for cyclic weak $\phi$-contraction," Applied Mathematics Letters, vol. 24, no. 6, pp. 822-825, 2011.
[4] E. Karapinar, M. Jleli, and B. Samet, "Fixed point results for almost generalized cyclic $(\Psi, \phi)$-weak contractive type mappings with applications," Abstract and Applied Analysis, vol. 2012, Article ID 917831, 17 pages, 2012.

[5] E. Karapinar and K. Sadarangani, "Fixed point theory for cyclic $(\varphi-\psi)$-contractions," Fixed Point Theory and Applications, vol. 2011, article 69, 2011.

[6] E. Karapinar and S. Moradi, "Fixed point theory for cyclic genetalized $(\phi, \phi)$-contraction mappings," Annali Dell'Universita' di Ferrara. In press.

[7] E. Karapinar and H. K. Nashine, "Fixed point theorem for cyclic weakly Chatterjea type contractions," Journal of Applied Mathematics, vol. 2012, Article ID 165698, 15 pages, 2012.

[8] E. Karapinar and H. K. Nashine, "Fixed point theorems for Kanaan type cyclic weakly contractions," Nonlinear Analysis and Optimization. In press.

[9] H. K. Nashine, "Cyclic generalized $\psi$-weakly contractive mappings and fixed point results with applications to integral equations," Nonlinear Analysis, vol. 75, no. 16, pp. 6160-6169, 2012.

[10] S. G. Matthews, "Partial metric topology," Annals of the New York Academy of Sciences, vol. 728, pp. 183-197, 1994, Proceedings of the 8th Summer Conference on General Topology and Applications.

[11] R. Heckmann, "Approximation of metric spaces by partial metric spaces," Applied Categorical Structures, vol. 7, no. 1-2, pp. 71-83, 1999.

[12] S. Oltra and O. Valero, "Banach's fixed point theorem for partial metric spaces," Rendiconti dell'Istituto di Matematica dell'Università di Trieste, vol. 36, no. 1-2, pp. 17-26, 2004.

[13] O. Valero, "On Banach fixed point theorems for partial metric spaces," Applied General Topology, vol. 6, no. 2, pp. 229-240, 2005.

[14] H.-P. A. Künzi, H. Pajoohesh, and M. P. Schellekens, "Partial quasi-metrics," Theoretical Computer Science, vol. 365, no. 3, pp. 237-246, 2006.

[15] M. Bukatin, R. Kopperman, S. Matthews, and H. Pajoohesh, "Partial metric spaces," American Mathematical Monthly, vol. 116, no. 8, pp. 708-718, 2009.

[16] S. Romaguera, "A Kirk type characterization of completeness for partial metric spaces," Fixed Point Theory and Applications, vol. 2010, Article ID 493298, 6 pages, 2010.

[17] D. Ilić, V. Pavlović, and V. Rakočević, "Some new extensions of Banach's contraction principle to partial metric space," Applied Mathematics Letters, vol. 24, no. 8, pp. 1326-1330, 2011.

[18] D. Ilić, V. Pavlović, and V. Rakočević, "Extensions of the Zamfirescu theorem to partial metric spaces," Mathematical and Computer Modelling, vol. 55, no. 3-4, pp. 801-809, 2012.

[19] E. Karapinar, "Generalizations of Caristi Kirk's theorem on partial metric spaces," Fixed Point Theory and Applications, vol. 2011, article 4, 2011.

[20] C. Di Bari, Z. Kadelburg, H. K. Nashine, and S. Radenović, "Common fixed points of g-quasicontractions and related mappings in 0-complete partial metric spaces," Fixed Point Theory and Applications, vol. 2010, article 113, 2012.

[21] K. P. Chi, E. Karapınar, and T. D. Thanh, "A generalized contraction principle in partial metric spaces," Mathematical and Computer Modelling, vol. 55, no. 5-6, pp. 1673-1681, 2012.

[22] L. Ćirić, B. Samet, H. Aydi, and C. Vetro, "Common fixed points of generalized contractions on partial metric spaces and an application," Applied Mathematics and Computation, vol. 218, no. 6, pp. 2398-2406, 2011. 
[23] E. Karapinar, "Weak $\phi$-contraction on partial metric spaces," Journal of Computational Analysis and Applications, vol. 14, no. 2, pp. 206-210, 2012.

[24] E. Karapınar and U. Yüksel, "Some common fixed point theorems in partial metric spaces," Journal of Applied Mathematics, vol. 2011, Article ID 263621, 16 pages, 2011.

[25] E. Karapinar, "A note on common fixed point theorems in partial metric spaces," Miskolc Mathematical Notes, vol. 12, no. 2, pp. 185-191, 2011.

[26] S. Romaguera, "Fixed point theorems for generalized contractions on partial metric spaces," Topology and Its Applications, vol. 159, no. 1, pp. 194-199, 2012.

[27] H. K. Nashine and E. Karapinar, "Fixed point results in orbitally complete partial metric spaces," Bulletin of the Malaysian Mathematical Sciences Society. In press.

[28] H. Aydi, "Fixed point theorems for generalized weakly contractive condition in ordered partial metric spaces," Journal of Nonlinear Analysis and Optimization, vol. 2, no. 2, pp. 269-284, 2011.

[29] H. Aydi, "Common fixed point results for mappings satusfying $(\Psi, \phi)$-weak contractions in ordered partial metric spaces," International Journal of Mathematics and Statistics, vol. 12, pp. 53-64, 2012.

[30] H. Aydi, E. Karapınar, and W. Shatanawi, "Coupled fixed point results for $(\psi, \varphi)$-weakly contractive condition in ordered partial metric spaces," Computers \& Mathematics with Applications, vol. 62, no. 12, pp. 4449-4460, 2011.

[31] S. Romaguera, "Matkowski's type theorems for generalized contractions on (ordered) partial metric spaces," Applied General Topology, vol. 12, no. 2, pp. 213-220, 2011.

[32] B. Samet, "Coupled fixed point theorems for a generalized Meir-Keeler contraction in partially ordered metric spaces," Nonlinear Analysis, vol. 72, no. 12, pp. 4508-4517, 2010.

[33] B. Samet, M. Rajović, R. Lazović, and R. Stoiljković, “Common fixed point results for nonlinear contractions in ordered partial metric spaces," Fixed Point Theory and Applications, vol. 2011, article 71, 2011.

[34] H. K. Nashine, Z. Kadelburg, and S. Radenović, "Common fixed point theorems for weakly isotone increasing mappings in ordered partial metric spaces," Mathematical and Computer Modelling. In press.

[35] H. K. Nashine, Z. Kadelburg, S. Radenović, and J. K. Kim, "Fixed point theorems under Hardy-Rogers weak contractive conditions on 0-complete ordered partial metric spaces," Fixed Point Theory and Applications, vol. 2012, article 180, 2012.

[36] D. Paesano and P. Vetro, "Suzuki's type characterizations of completeness for partial metric spaces and fixed points for partially ordered metric spaces," Topology and Its Applications, vol. 159, no. 3, pp. 911-920, 2012.

[37] C. Di Bari and P. Vetro, "Fixed points for weak $\phi$-contractions on partial metric spaces," International Journal of Contemporary Mathematical Sciences, vol. 1, pp. 5-12, 2011.

[38] M. Abbas, T. Nazir, and S. Romaguera, "Fixed point results for generalized cyclic contraction mappings in partial metric spaces," Revista de la Real Academia de Ciencias Exactas, Físicas y Naturales A, vol. 106, pp. 287-297, 2012.

[39] R. P. Agarwal, M. A. Alghamdi, and N. Shahzad, "Fixed point theory for cyclic generalized contractions in partial metric spaces," Fixed Point Theory and Applications, vol. 2012, article 40, 2012.
[40] E. Karapinar and I. S. Yuce, "Fixed point theory for cyclic generalized weak $\varphi$-contraction on partial metric spaces," Abstract and Applied Analysis, vol. 2012, Article ID 491542, 12 pages, 2012.

[41] E. Karapinar, N. Shobkolaei, S. Sedghi, and S. M. Vaezpour, "A common fixed point theorem for cyclic operators in partial metric spaces," Filomat, vol. 26, pp. 407-414, 2012.

[42] M. S. Khan, M. Swaleh, and S. Sessa, "Fixed point theorems by altering distances between the points," Bulletin of the Australian Mathematical Society, vol. 30, no. 1, pp. 1-9, 1984.

[43] B. S. Choudhury, "Unique fixed point theorem for weak Ccontractive mappings," Kathmandu University Journal of Science, Engineering and Technology, vol. 5, pp. 6-13, 2009.

[44] A. G. B. Ahmad, Z. M. Fadail, H. K. Nashine, Z. Kadelburg, and $S$. Radenović, "Some new common fixed point results through generalized altering distances on partial metric spaces," Fixed Point Theory and Applications, vol. 2012, article 120, 2012.

[45] H. Aydi, "A common fixed point result by altering distances involving a contractive condition of integral type in partial metric spaces," Demonstratio Mathematica. In press.

[46] T. Abdeljawad, E. Karapınar, and K. Taş, "Existence and uniqueness of a common fixed point on partial metric spaces," Applied Mathematics Letters, vol. 24, no. 11, pp. 1900-1904, 2011.

[47] E. Karapinar and I. M. Erhan, "Fixed point theorems for operators on partial metric spaces," Applied Mathematics Letters, vol. 24, no. 11, pp. 1894-1899, 2011.

[48] M. A. Petric, "Best proximity point theorems for weak cyclic Kannan contractions," Filomat, vol. 25, no. 1, pp. 145-154, 2011. 


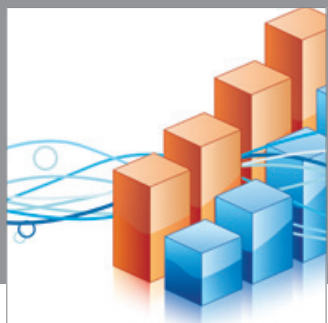

Advances in

Operations Research

mansans

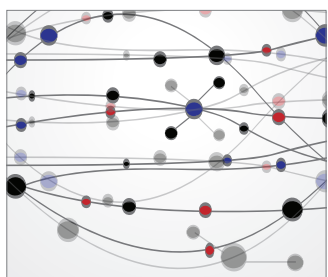

The Scientific World Journal
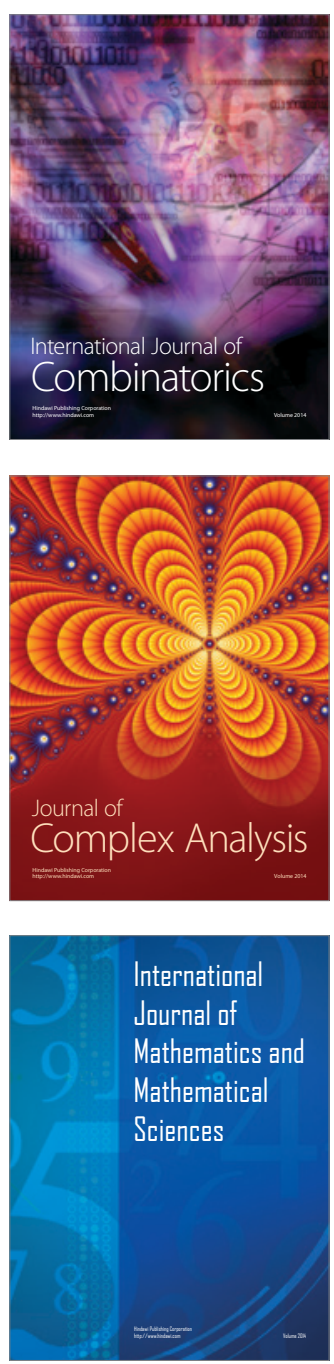
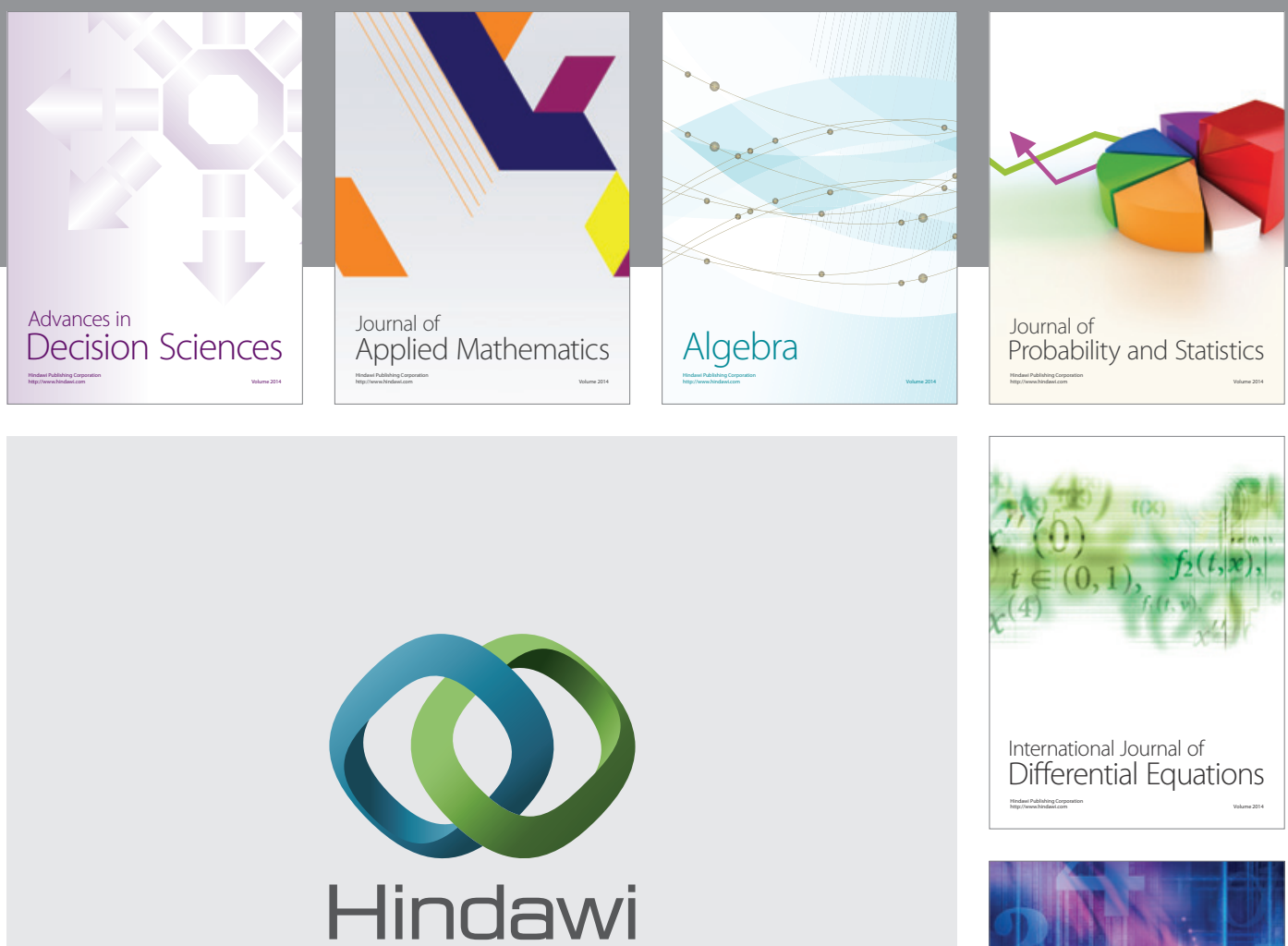

Submit your manuscripts at http://www.hindawi.com
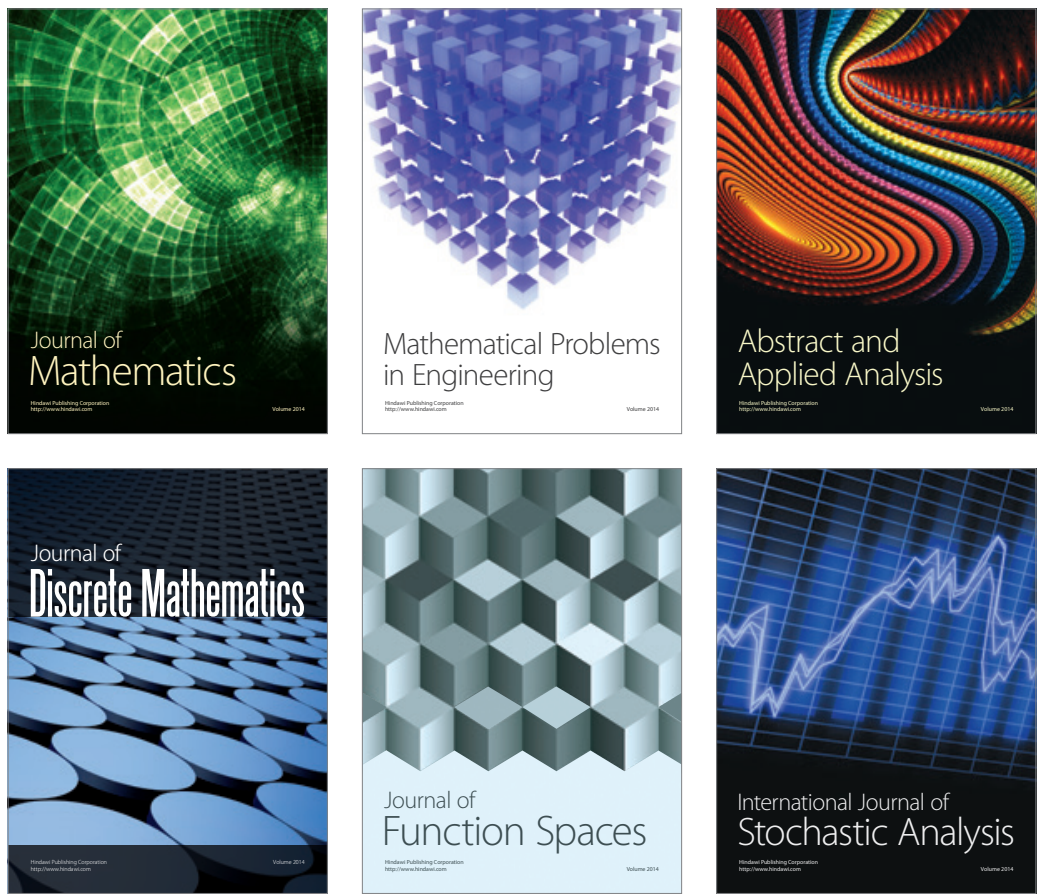

Journal of

Function Spaces

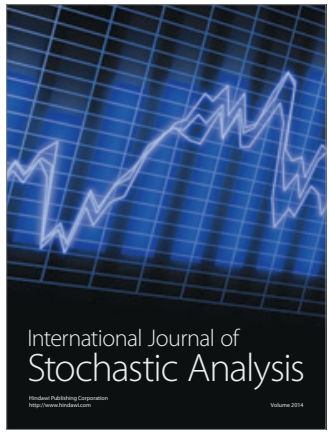

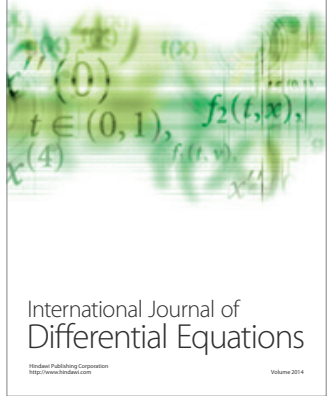
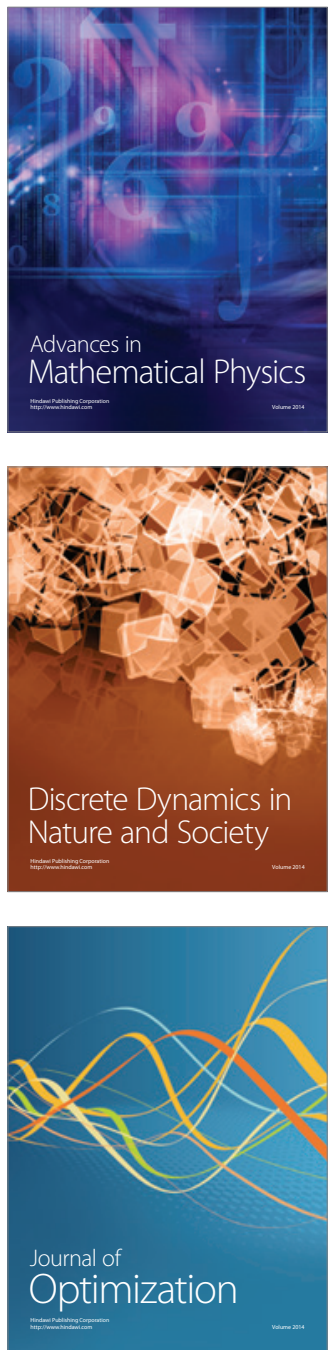